\title{
NONLINEAR FORCE TRACKING CONTROL OF ELECTROHYDROSTATIC ACTUATORS SUBMITTED TO MOTION DISTURBANCES
}

\author{
Tahereh Vaezi*, Mohamed Smaoui, Paolo Massioni, Xavier Brun, Eric Bideaux \\ Laboratoire Ampère CNRS, INSA Lyon, université de Lyon,France \\ * Corresponding author: E-mail address: Tahereh.vaezi@insa-lyon.fr
}

\begin{abstract}
In some industrial fields, such as aerospace, electro-hydrostatic actuators (EHAs) are increasingly used to replace conventional standard hydraulic actuators due to their better energy performance. Moreover, implementing different type or technology of actuators in redundant actuation systems working on the same moving part introduced some new challenges. This paper presents a force-tracking controller for an asymmetric electro-hydrostatic actuator that is submitted to an external motion generated by an external source. In this case, the rod displacement is considered as an external disturbance for the hydraulic cylinder, but it is assumed that this disturbance can be easily measured using sensors. The theoretical motivation of this work is discussed along and a variable gain state feedback control based on Linear Parameter Varying control (LPV) theory is proposed to achieve stability, disturbance rejection and tracking performance. The Linear Matrix Inequalities (LMI) framework is used to determine a control law including an augmented state feedback with an integral action that reduces trajectory-tracking errors. Simulation results of the control law are finally given to verify the global performance of this control design.
\end{abstract}

Keywords: electro hydrostatic actuator, force control, Linear parameter variant.

\section{INTRODUCTION}

Moving towards more electrical systems is generating significant efforts to develop electric powered actuators, especially in aeronautics and automotive industries. For such applications, it may be advantageous to combine a conventional hydraulic actuator and an electric power source. Nowadays, electro-hydrostatic actuators (EHA) have been considered in modern industries due to their reliability, durability, high energetic efficiency and less losses of energy. However, EHAs are highly nonlinear and complex systems. Nonlinearities are mainly related to hydraulic phenomena during the pushing and pulling stroke of the asymmetric cylinder such as variations of the fluid volume under compression, flow characteristics of orifices and pipes, and seal friction. Besides the nonlinearities, some uncertainties originate from fluctuation in supplied pump flow or variation of some parameters such as the bulk modulus and the fluid viscosity. All these considerations make the design of efficient controllers for EHAs really challenging.

Most of the previous research works on force control in the case of hydraulic actuators consider that the rod is fixed or that its displacement is negligible. With this assumption, the volumes of the chambers are generally constant and the flow rate corresponds to the compressibility flow. The control synthesis is consequently simplified.

The literature review brings out different control techniques to solve the force control problem, especially for servo controlled hydraulic actuators (SHA). Among these, PID, lead-lag controllers $[1,2]$, quantitative feedback theory (QFT) method [3, 4], self-tuning QFT control [5], feed-forward inverse model control [6], fractional order controller [7], hybrid fuzzyneural technique [8], nonlinear control algorithms $[9,10,11]$ and robust $H_{\infty}$ control [12] have been applied. In these researches, some do not consider the inherent nonlinearities, friction, etc., and some are not practical. Predominantly, in most of these papers, first the effect of the rod 
displacement is not taken into account or neglected, and second the stability of the inner states is not discussed. Beside, hydraulic architecture based on independent inputs have been proposed $[13,14,15]$, this enables to simultaneously control the effort and the displacement. In this case, thanks to the two inputs the system stability is insured in the whole range of operating points.

Therefore, the objective of this work is to analyze and design of a proper controller for the force trajectory tracking when the EHA is submitted to an external motion. The novelty is here to use a single pump in order to achieve force trajectory tracking. Consequently, difficulties arise since only one input is available and the motion (disturbance) can be antagonist to the force trajectory. Moreover, due to the fact that movement is considered as a disturbance, the EHA is just modeled by its hydraulic equations, which can be considered as a linear parameter varying (LPV) model as it will be shown in section 3 .

The study of LPV systems is motivated by the gain scheduling control design methodology. LPV control theory is advantageous because it provides a generic control synthesis method, which insures stability and performance over a wide range of parameter variation. In general, the solution to the LPV control analysis and synthesis problems is formulated as a parameter-dependent linear matrix inequalities (LMIs) problem, which corresponds to a special type of convex optimization problem. The LMI approach provides the design framework to determine feedback laws aiming at asymptotic stabilization including $H_{\infty}$ and robustness features [16, 17].

In this research, we will apply this method to solve numerically parameter-dependent LMIs associated with LPV analysis and synthesis in the case of the EHA force control. A state feedback control will be deduced by a direct application of Sum Of Squares (SOS) decompositions to the Lyapunov stability analysis for the LPV model.

The remaining sections are organized as follows: first, in Section 2, we will start with basic background of LMI control theory and the formulation needed to solve the SOS problem. In Section 3, we will introduce the model of the system. Then, the LPV control model of the EHA submitted to an external motion will be formulated. In Section 4, we will design the integral state feedback controller based on introduced design procedure for an augmented model of the EHA. In section 5, before concluding, simulation results of closed loop system will be carried out to demonstrate the proposed control method and investigate the effectiveness and robustness of the proposed controller. The capability of the proposed controller will be compared with a controller with parameter-independent gains for the force tracking trajectory.

\section{LMI CONTROL THEORY}

This section presents the key concept of the control method proposed in this paper. First, we will introduce the basic of Lyapunov stability with the LMI framework. Next, we will formulate the LMI, in the form of Sum Of Squares (SOS). These concepts will be applied in Section 4 to derive a state feedback controller for an EHA submitted to an external motion.

First, let us consider a representation of a LPV system as:

$$
\left\{\begin{array}{c}
\dot{x}(t)=A(\rho(t)) x(t)+B_{u}(\rho(t)) u(t)+\cdots \\
\quad \ldots+B_{w}(\rho(t)) w(t) \\
y(t)=C(\rho(t)) x(t)
\end{array}\right.
$$

where $x(t)$ is the state vector, $u(t)$ is the control input, $w(t)$ is the exogenous inputs such as perturbation and $y(t)$ is the output of the system.

Assumption1. The state vector $x(t)$ is measurable or can be estimated online.

Assumption2. $\rho(t)$ and $\dot{\rho}(t)$ are continuous and bounded functions of t ( hereafter $\rho$ will be used instead of $\rho(t)$ ).

Lyapunov theory states that the existence of a matrix $P(\rho)$ such that the quadratic Lyapunov function $V(x)$

$$
V(x)=x^{T} P(\rho) x>0 \text { for all } x \neq 0
$$

satisfies:

$$
\dot{V}(x)=\dot{x}^{T} P(\rho) x+x^{T} P(\rho) \dot{x}+x^{T} \dot{P}(\rho) x<0
$$

is a necessary and sufficient condition to ensure stability of the system. Equation (2) is a LMI and $P(\rho)$ can be found by solving these inequalities. 
Consider system (1) in closed loop with a state feedback controller, $u=K(\rho) x$. From (1), the closed loop model becomes:

$$
\left\{\begin{array}{l}
\dot{x}=\left(A(\rho)+B_{u}(\rho) K(\rho)\right) x+B_{w}(\rho) w \\
y=C(\rho(t)) x
\end{array}\right.
$$

Furthermore, if a $\mathcal{L}_{2}$ gain $\gamma$ constraint for disturbance rejection (5) is added to the previous stability objective, the problem can be expressed similarly on finding a feedback $K(\rho)$ that satisfies the LMIs (2), (3) and (5).

$$
\frac{\|y\|_{2}}{\|w\|_{2}} \leq \gamma, \quad \gamma>0
$$

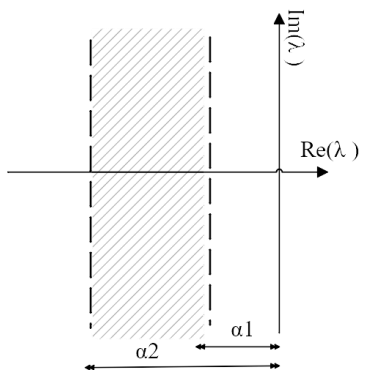

Figure 1: Acceptable pole placement area

However, high controller gains can lead system to instability, due to the discrete-time implementation of the control law. In order to avoid this problem, an upper bound for the module of poles, $\alpha_{2}$, should be chosen for pole placement. Therefore, to guarantee prescribed performance requirements, the closed-loop pole locations must be restricted into a specific region (Figure 1). Equations (6) then define equivalent Lyapunov conditions.

$\left\{\begin{array}{l}V(x)>0 \\ \dot{V}(x)<-\alpha_{1} V(x) \\ \dot{V}(x)>-\alpha_{2} V(x)\end{array}\right.$

The following theorem [19] presents the gain controller design using regional pole location constraints.

Theorem 1: Consider the LPV system in closedloop with a state feedback controller $(u=$ $\left.K(\rho) x=L(\rho) X^{-1}(\rho) x\right)$, as given in (4). The closed-loop system is stable with an $L_{2}$ gain less than $\gamma>0$, with its closed-loop poles in the specified area if there exists a positive definite matrix $X(\rho) \in \mathfrak{R}^{n \times n}$ and a rectangular matrix $L(\rho) \in \mathfrak{R}^{m \times n}$ subject to (7-9), where $X(\rho)=$ $P^{-1}(\rho)$ and $L(\rho)=K(\rho) X(\rho)$.

If such problem is feasible, a suitable controller gain is:

$K(\rho)=L(\rho) X^{-1}(\rho)$.

Notice that theorem 1 features parameter dependent LMIs, as explained in [20]. These can be solved efficiently (convex optimisation) by the so-called SOS technique [21].

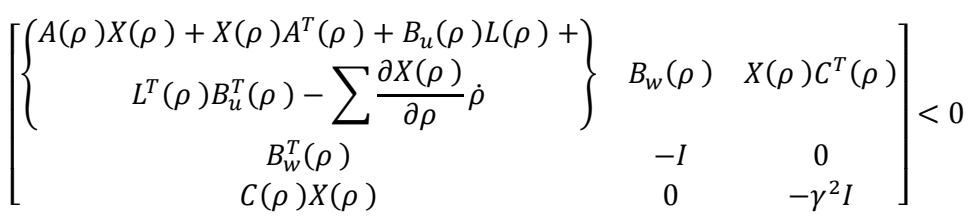

$$
\begin{aligned}
& A(\rho) X(\rho)+X(\rho) A^{T}(\rho)+B_{u}(\rho) L(\rho)+L^{T}(\rho) B_{u}^{T}(\rho)-\sum \frac{\partial X(\rho)}{\partial \rho} \dot{\rho}<-2 \alpha_{1} X(\rho) \\
& A(\rho) X(\rho)+X(\rho) A^{T}(\rho)+B_{u}(\rho) L(\rho)+L^{T}(\rho) B_{u}^{T}(\rho)-\sum \frac{\partial X(\rho)}{\partial \rho} \dot{\rho}>-2 \alpha_{2} X(\rho)
\end{aligned}
$$

Definition of SOS: $X$ is positive if $X(\rho)>0$ for all $\in \mathcal{R}^{n \times n}$. A way of establishing whether a function is positive consists of establishing whether it can be written as a Sum Of Squares (SOS) of polynomials. 


$$
X(\rho)=\sum X_{i}^{2}(\rho)
$$

Implementing SOS decomposition is interested because establishing whether a polynomial is SOS, leads to solving a convex optimization problem. Being stricter, the condition that $X(\rho)$ is SOS is computationally much more tractable than positivity; therefore, positivity condition of Lyapunov function can be replaced by the SOS condition.

Theorem 2: [22] Consider system (1). Suppose that there exist a symmetric polynomial matrix $X$ and, a polynomial matrix $L$, a parameter $\varepsilon_{1}>0$ and an SOS polynomial $\varepsilon_{2}: \mathcal{R}^{n} \rightarrow \Re$ such that

$$
\begin{array}{ll}
- & X(\rho)-\varepsilon_{1} I>0 \quad \text { is SOS } \\
- & -\left(A^{T}(\rho) X(\rho)+X(\rho) A(\rho)+\right. \\
& L^{T}(\rho) B^{T}(\rho)+B(\rho) L(\rho)-\sum \frac{\partial X}{\partial \rho} \dot{\rho}+ \\
& \left.\varepsilon_{2} I\right)>0 \quad \text { is SOS }
\end{array}
$$

Then, the origin of (1) is asymptotically stabilized by a state feedback given by (10).

This technique is originally proposed for polynomial systems, but thanks to some new researches [23], it has been extended to nonpolynomial systems.

\section{MODELLING}

The test rig at which this study is applied is shown in Figure 2. It is a redundant system composed of an EHA connected directly to an electromechanical actuator (EMA). The EMA is position controlled, that is why it will be considered here as the external source generating motion trajectory. On the EHA side, several components are implemented, such as safety valves, electrical motor, hydraulic pump, sensors, etc. Beside the effect of the main components, the performance of the EHA is also affected by pressure losses in transmission lines, parameter changes during the working time, due to temperature change for example.

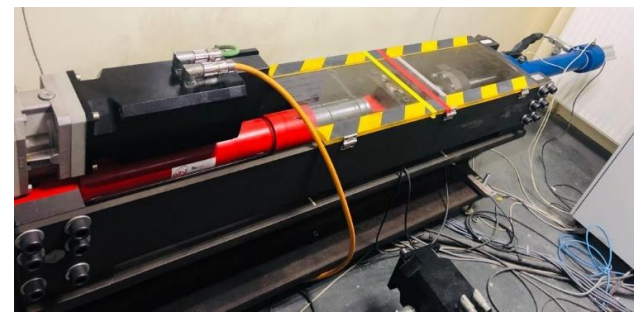

Figure 2: EHA-EMA test rig

There are three different possible configurations of an EHA: Fixed Pump displacement and Variable Motor speed (FPVM), Variable Pump displacement and Fixed Motor speed (VPFM) and Variable Pump displacement and Variable Motor speed (VPVM). Our test rig is equipped with a VPVM configuration and enables therefore to consider the different ways of generating flow

\subsection{Simulation Model of EHA}

The simulation model has been defined to cross over the gap between control model and reality. It takes into account all the main components of the hydraulic parts and mostly based on physical characteristics provided by datasheets or direct measurements on the test rig. However, some hypothesis have been considered to avoid useless complexity:

- The hydraulic transmission lines are short with reasonably large diameter to keep fluid flow velocities to a low value. In these situations, it is usually reasonable to ignore line resistance, relative line capacitance and fluid inertia, control valve resistance and the inertia of the actuator and load.

- The inlet/outlet port pressures of the hydraulic pump are regarded equal to the pressures inside the chambers of the cylinder.

- The behaviour of the system is supposed to be isothermal.

- The pump/motor unit is an ideal flow rate unit.

The simulation model has been developed in the Simcenter Amesim software from Siemens as shown in Figure 3. 


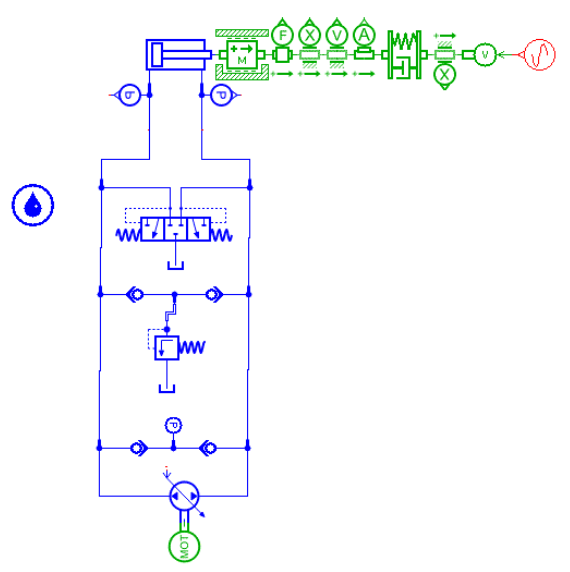

Figure 3: Simulation model in Simcenter Amesim

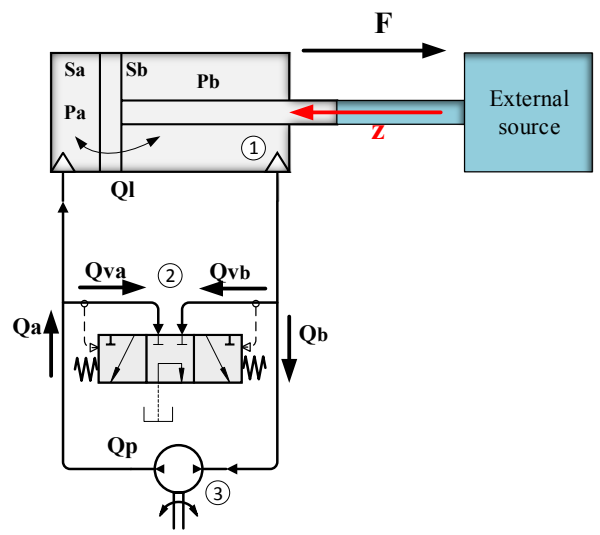

Figure 4: Scheme of the control model

\subsection{Control Model of EHA}

A control model of the EHA (Figure 4) is deduced in this section with the following additional assumptions:

- Dynamic of the pump is neglected. (Figure 4-3 )

- The recirculation valve is considered to have a linear characteristic and its dynamic is neglected. (Figure 4-2 )

- Safety and bypass valves are not modelled.

- Minimum pressure of the tank is consider to be zero.

As it is emphasized in Figure 4, the cylinder position, $z$, is negative when the cylinder extends, and positive otherwise, and the force, $F$, is positive if it is oriented against the velocity.
The flow rate equations of the chambers can be obtained as the following expressions:

$$
\begin{aligned}
& Q_{a}=S_{a} \dot{z}+\frac{V_{a}}{\beta} \dot{P}_{a}+Q_{l}+Q_{v a} \\
& Q_{b}=S_{b} \dot{z}-\frac{V_{b}}{\beta} \dot{P}_{b}+Q_{l}-Q_{v b}
\end{aligned}
$$

where, $P_{a}, P_{b}$ are respectively the pressures in the cap side and rod side, and:

$$
\begin{aligned}
& V_{a}=V_{0 a}+S_{a} Z \\
& V_{b}=V_{0 b}-S_{b} Z
\end{aligned}
$$

$V_{a}, V_{b}$ are volumes in each side of the actuator, $V_{0 a}, V_{0 b}$ are dead volumes and $Q_{v a}$ and $Q_{v b}$ represent the flow rates due to the recirculation valve that can be expressed with:

$$
\begin{aligned}
& Q_{v i}=K_{v i} P_{i}, i=a, b \\
& \left\{\begin{array}{l}
K_{v b}=0 \text { if } P_{b}>P_{a} \\
K_{v a}=0 \text { if } P_{a}>P_{b}
\end{array}\right.
\end{aligned}
$$

Finally, $Q_{l}$ is the linear flow leakage between the chambers:

$$
Q_{l}=K_{f}\left(P_{a}-P_{b}\right)
$$

The output, here the force applied by the hydraulic actuator on the moving mass, is a combination of the hydraulic force and mechanical, inertia and friction forces which are considered negligible compared to the hydraulic force, thus the output is expressed as:

$$
F=S_{a} P_{a}-S_{b} P_{b}
$$

The effect of the transmission lines are neglected. So, the flows $Q_{P}, Q_{a}$ and $Q_{b}$ are equal and a function of pump displacement and motor speed (18). Moreover, the VPVM configuration enables the flow rate of the hydraulic pump then to be expressed by the following equation:

$$
Q_{P}=D_{\max } \omega_{\max } u=Q_{\max } u
$$

where $\omega_{\max }$ is the maximum motor speed, $D_{\max }$ is the maximum hydraulic pump displacement and $u \in\left[\begin{array}{ll}-1 & 1\end{array}\right]$ is the percentage of the maximum flow rate $\left(Q_{\max }\right)$ which will be taken as the command input. Let us define yet the following change of variable: $\rho=z, w=\dot{z}, x=$ $\left[\begin{array}{ll}P a & P_{b}\end{array}\right]^{T}, y=F$.

The LPV model of the EHA is then expressed as:

$\left\{\begin{array}{l}\dot{x}=A(\rho) x+B_{u}(\rho) u+B_{w}(\rho) w \\ y=C x\end{array}\right.$ 


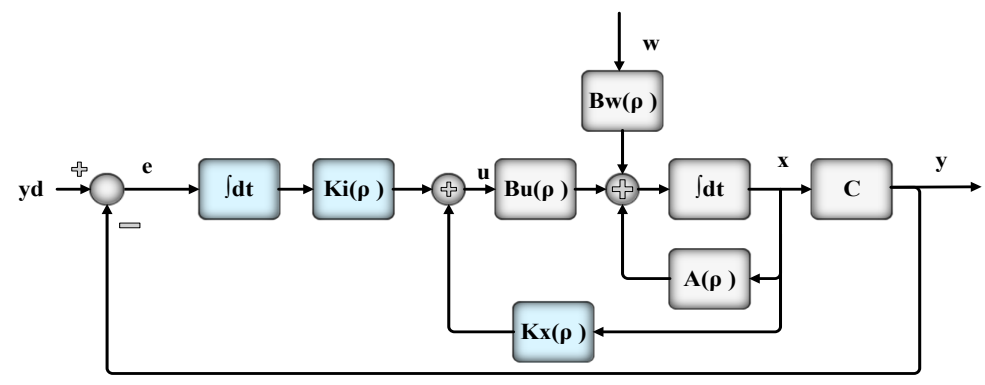

Figure 5: Closed-loop scheme of the system

With:

$$
\left\{\begin{array}{l}
A=\left[\begin{array}{cc}
\frac{-\beta\left(K_{v a}+K_{f}\right)}{V_{a}(\rho)} & \frac{\beta K_{f}}{V_{a}(\rho)} \\
\frac{\beta K_{f}}{V_{b}(\rho)} & \frac{-\beta\left(K_{v b}+K_{f}\right)}{V_{b}(\rho)}
\end{array}\right] \\
B_{u}=\left[\begin{array}{l}
\frac{\beta D_{\max } \omega_{n}}{V_{a}(\rho)} \\
\frac{-\beta D_{\max } \omega_{n}}{V_{b}(\rho)}
\end{array}\right], B_{w}=\left[\begin{array}{l}
\frac{-\beta S_{A}}{V_{a}(\rho)} \\
\frac{\beta S_{B}}{V_{b}(\rho)}
\end{array}\right] \\
C=\left[\begin{array}{ll}
S_{a} & \left.-S_{b}\right]
\end{array}\right]
\end{array}\right.
$$

Notice that, the control model involves two continuous dynamic models depending on the pressures $P_{a}$ and $P_{b}$ (15), which are both controllable and marginally stable for all $\rho \leq$ $\rho(t) \leq \bar{\rho}$ but uncontrollable model for $P_{a}=\bar{P}_{b}$.

\section{CONTROLLER SYNTHESIS}

In this section, a parameter dependent statefeedback controller is designed for the force tracking of the EHA submitted to an important external motion. In order to compensate model uncertainties and achieving zero steady state error, an integral action is added to the state feedback controller. Figure 5 shows the scheme of the control including the state feedback and the integral action. Therefore, let us add in the state space model (19-20) an extra state, $x_{i}$, that is the integral of the output error:

$$
x_{i}=\int\left(y_{d}-y\right) d t=\int\left(y_{d}-C x\right) d t
$$

with $x_{a}=\left[\begin{array}{ll}x^{T} & x_{i}\end{array}\right]^{T}$ the augmented state vector, $w_{a}=\left[\begin{array}{ll}w & y_{d}\end{array}\right]^{T}$ the augmented exogenous vector and $C_{a}=\left[\begin{array}{ll}C & 0\end{array}\right]$ the augmented output vector and:

$$
\begin{aligned}
& A_{a}(\rho)=\left[\begin{array}{cc}
A_{2 \times 2} & 0_{2 \times 1} \\
-C_{1 \times 2} & 0
\end{array}\right], \\
& B_{a_{u}}=\left[\begin{array}{c}
B_{u_{2 \times 2}} \\
0
\end{array}\right], B_{a_{w}}=\left[\begin{array}{cc}
B_{w_{2 \times 1}} & 0_{2 \times 1} \\
0 & 1
\end{array}\right]
\end{aligned}
$$

Let us now introduce the augmented model as a linear model with a scalar scheduling parameter:

$$
\begin{aligned}
& A_{a}=\Delta^{-1}(\rho)\left(\hat{A}_{0}+\hat{A}_{1} \rho+\hat{A}_{2} \rho^{2}\right) \\
& B_{a_{u}}=\Delta^{-1}(\rho)\left(\hat{B}_{u_{0}}+\hat{B}_{u_{1}} \rho+\hat{B}_{u_{2}} \rho^{2}\right) \\
& B_{a_{w}}=\Delta^{-1}(\rho)\left(\hat{B}_{w_{0}}+\hat{B}_{w_{1}} \rho+\hat{B}_{w_{2}} \rho^{2}\right)
\end{aligned}
$$

where $\hat{A}_{i}, \hat{B}_{u_{i}}$ and $\hat{B}_{w_{i}}(i=1,2,3)$ are constant matrices of the appropriate dimension and $\Delta(\rho)$ is a polynomial factor that is always positive:

$\Delta(\rho)=\frac{V_{a}(\rho) V_{b}(\rho)}{V_{a 0} V_{b 0}}$

Theorem 1 is applied to find the controller gains. Considering that $X(\rho)$ and $L(\rho)$ are chosen to be respectively a constant matrix and a linear polynomial vector defined by now:

$X=X_{0}$

$L=L_{0}+L_{1} \rho$

Therefore, $\partial X / \partial \rho=0$ and it is eliminated from (7-9). The controller gains, $K(\rho)=$ $\left[K_{x}(\rho) \quad K_{i}(\rho)\right]$, with the $\mathcal{L}_{2}$ gain bound $\gamma=1$ are calculated using the Sedumi solver. The LPV controller gains are then derived thanks to (10)

The earlier discussion explained that recirculation valve would provide different models and each model requests its own controller to overcome disturbances and ensure closed loop performance requirements. Therefore, employing switch between controllers is an essential issue. Moreover, stability of the whole system is guaranteed for $P_{a}<P_{b}$ (or $P_{a}>$ $\left.P_{b}\right)$. However, switches will happen in pressure equalities (uncontrollable modes) where 
establishing stability of whole system is a complicated problem, which is not discussed here.

\section{SIMULATION RESULTS}

The goal of this section is to demonstrate feasibility of the LMI force controller and to illustrate the capability of the proposed approach for disturbance rejection in tracking force trajectory.

Therefore, the controller is executed on the simulation model with the hypothesis introduced in section 3.1. Simcenter Amesim software from Siemens (see Figure 3) is used to examine the suggested controller. System parameters are given in table 1 .

The ability of the controlled system to follow a sine-wave trajectory, $y_{d}=F_{0} \sin (2 \pi t)+F_{1}$, with $F_{0}=7 \mathrm{kN}$ and $F_{1}=2 \mathrm{kN}$ is investigated in Figure 7, whereas the rod is submitted to different sinusoidal motions generated by the external source (Figure 6).

Table 1: Parameters of the EHA

\begin{tabular}{|l|l|l|}
\hline Cylinder parameter & Value & unit \\
\hline Cap end area $\mathrm{S}_{\mathrm{a}}$ & 0.0032 & $\mathrm{~m}^{2}$ \\
\hline Rod end area $\mathrm{S}_{\mathrm{b}}$ & 0.0019 & $\mathrm{~m}^{2}$ \\
\hline bulk modulus $\beta$ & 1700 & $\mathrm{MPa}$ \\
\hline Inactive volume $V_{a 0}$ & $0.649 \times 10^{-3}$ & $\mathrm{~m}^{3}$ \\
\hline Inactive volume $V_{b 0}$ & $0.387 \times 10^{-3}$ & $\mathrm{~m}^{3}$ \\
\hline $\begin{array}{l}\text { valve coefficient } \\
K_{v a}, K_{v b}\end{array}$ & 0.045 & $\mathrm{~L} / \mathrm{s} / \mathrm{bar}$ \\
\hline
\end{tabular}

Referring to Figure 7, the supplied force by the hydraulic actuator converged to the desired trajectory and the system showed good tracking performance and disturbance rejection for both imposed displacements.

The simulation results of the pressures in the chambers are shown in Figure 8, which shows that the responses contain oscillations around pressure equalities due to the action of the recirculation valve. In addition, it can be observed that the pressures are bound by the lower value defined by the tank.

However, for the force and displacement trajectories, the recirculation valve is always fully opened on the rod side for positive forces and on the cap side for negative forces. The related pressure of the chamber is therefore equal to the tank pressure, which means that the force is always affected by a single pressure.

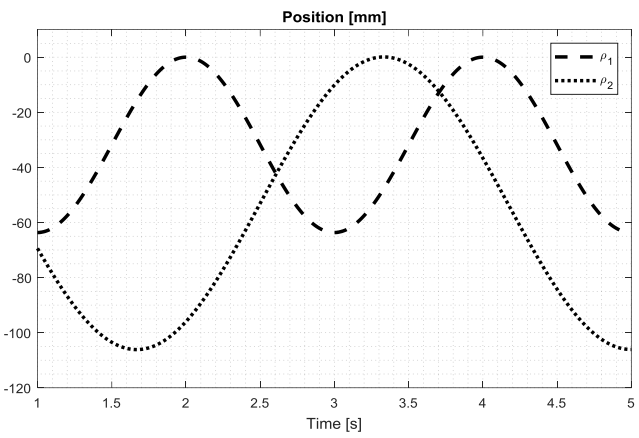

Figure 6: Imposed displacement trajectories

Input saturation may cause oscillatory transient behavior or even instability, and a closer inspection of the control input is shown in Figure 9. Here, as demonstrated, the control input is not saturated and therefore the oscillation of the pressures in Figure 8 can be mainly due to uncontrollability of the system while $P_{a}=P_{b}$.

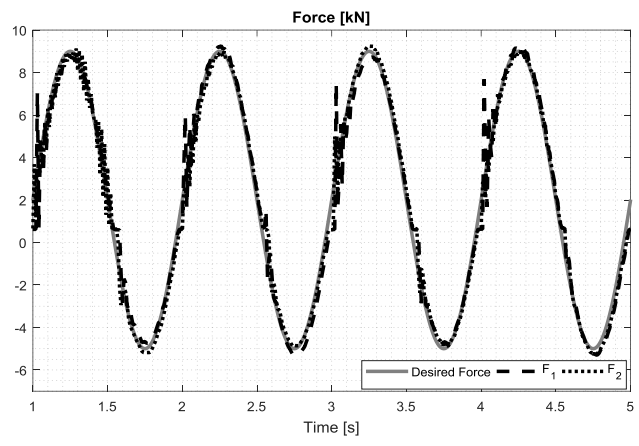

Figure 7: Simulation results for tracking desired force trajectory

Figure 10 shows the results obtained from the controller gains, as shown in the figure switches, which depend on the pressures $P_{a}$ and $P_{b}$. In addition, $K_{x}\left(b a r^{-1}\right)$ and $K_{x i}\left(N^{-1} s^{-1}\right)$ are the polynomial of displacement $(\rho(t)$ or $z(t))$. Although the variation of the gains is small, it is shown that good tracking performance cannot be achieved by constant gains, even for small displacements. 


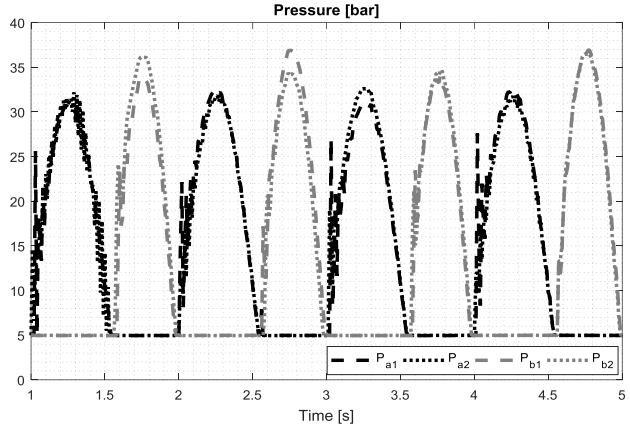

Figure 8: Simulation results for pressures

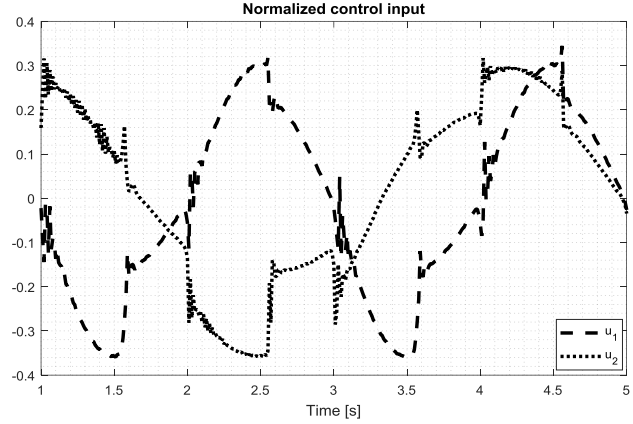

Figure 9: Control inputs

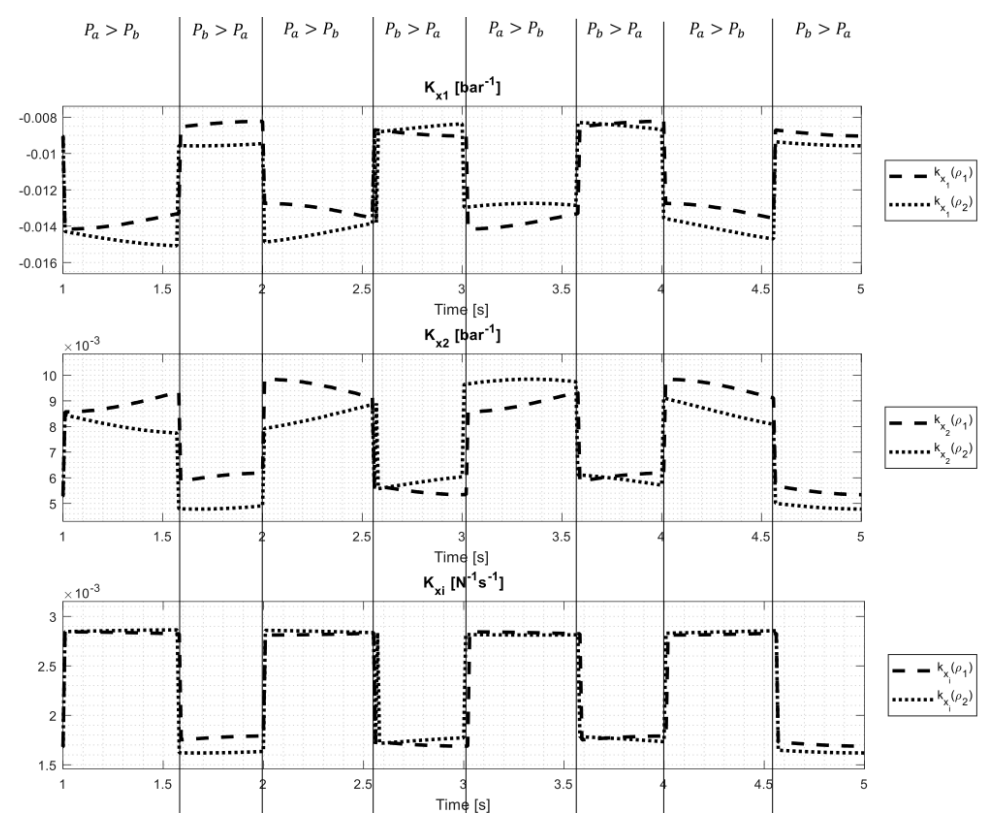

Figure 10: Simulation results for gains: a) $k_{x 1}$, b) $k_{x 2}$, c) $k_{x i}$

The force tracking performance of the system submitted to a small amplitude sinusoidal motion $(16 \mathrm{~mm})$ is shown in Figure 11. Clearly, the simulation results of the proposed approach (dash curve) presents better tracking performance properties compared to a state feedback controller with constant gains (dots curve).

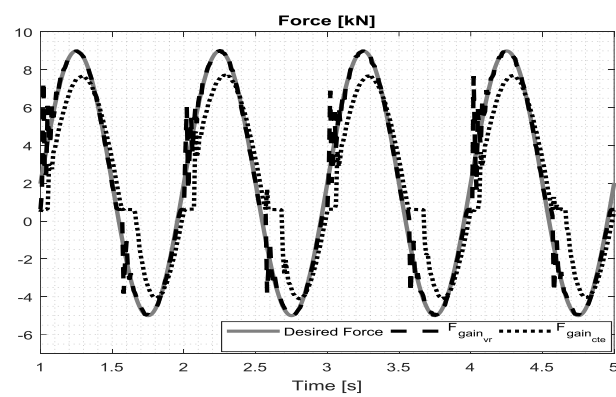

Figure 11: Tracking force trajectory by variable and constant gain controllers 
According to the carried out results, it can be concluded that the proposed controller improves the performance of the force control of the EHA when submitted to an external motion compared to state feedback controller with constant gains. In addition, the proposed controller guarantees significant disturbance rejection.

\section{CONCLUSION}

Due to the need for improved technology, industries have begun to use heterogeneous redundant actuation systems on their existing machines, leading to new challenges. Even though hydraulic power has always been recognized as being capable of applying high force, today it is necessary to overcome the problems caused by the force-fighting phenomenon that appears on redundant actuation systems.

This paper addressed a new controller for an electro hydrostatic actuator, which had been submitted to an external motion. First, a linear parameter variant model has been deduced. Second, a state-feedback controller with parameter-variant gains such as integral action has been established on the basis of Linear Matrix Inequalities framework and the Sum Of Squares (SOS) decomposition. The feasible solution of the proposed controller design procedure guarantees the Lyapunov stability and the perturbation rejection. Finally, the ability of the designed controller to follow the desired force trajectory has been verified by the simulation. The designed gains of the controller gains depend only on the position that can be easily measured by the sensors.

\section{NOMENCLATURE}

$\begin{array}{ll}S_{a} & \text { Cap end Area } \\ S_{b} & \text { Rod end Area } \\ V_{a 0} & \text { Inactive volume cap side } \\ V_{b 0} & \text { Inactive volume rod side } \\ \beta & \text { Bulk modulus } \\ P_{a}, x_{1} & \text { Pressure in cap side } \\ P_{b}, x_{2} & \text { Pressure in rod side } \\ P_{T} & \text { Tank pressure } \\ K_{f} & \text { Inter chamber leakage coefficient } \\ K_{v a}, K_{v b} & \text { Recirculation valve coefficient } \\ z, \rho & \text { Displacement of the cylinder } \\ w & \text { Velocity of the cylinder }\end{array}$

$\begin{array}{ll}F, y & \text { Force } \\ y_{d} & \text { Desired Force } \\ u & \text { Control input } \\ D_{\max } & \text { Hydraulic pump displacement } \\ \omega_{n} & \text { Nominal speed of electrical motor } \\ K(\rho) & \text { Controller gains } \\ Q_{p} & \text { Pump flowrate } \\ Q_{a} & \text { Transmission line flow rate } \\ Q_{b} & \text { Transmission line flow rate } \\ Q_{l} & \text { leakage flowrate } \\ Q_{v a}, Q_{v b} & \text { Recirculation valve flow rate } \\ E H A & \text { Electro hydrostatic actuator } \\ S H A & \text { servo controlled hydraulic actuators } \\ E M A & \text { Electromechanical actuator } \\ L P V & \text { Linear parameter variant } \\ L M I & \text { Linear matrix inequality } \\ S O S & \text { Sum Of Squares }\end{array}$

\section{REFERENCES}

[1] Alleyne, A. and Liu, R., (1999). On the limitations of force tracking control for hydraulic servosystems. Journal of dynamic systems, measurement, and control, 121(2), pp.184-190.

[2] Alleyne, A. and Liu, R., (2000). A simplified approach to force control for electro-hydraulic systems. Control Engineering Practice, 8(12), pp.1347-1356

[3] Niksefat, N. and Sepehri, N., (1999). Robust force controller design for an electrohydraulic actuator based on nonlinear model. In Proceedings 1999 IEEE International Conference on Robotics and Automation (Cat. No. 99CH36288C) (Vol. 1, pp. 200-206). IEEE.

[4] Niksefat, N. and Sepehri, N., (2001). Designing robust force control of hydraulic actuators despite system and environmental uncertainties. IEEE Control Systems Magazine, 21(2), pp.6677.

[5] Ahn, K.K. and Dinh, Q.T., (2009). Self-tuning of quantitative feedback theory for force control of an electro-hydraulic test machine. Control Engineering Practice, 17(11), pp.1291-1306.

[6] Zhao, J., Shen, G., Zhu, W., Yang, C. and Yao, J., (2016). Robust force control with a feed-forward inverse model controller for electro-hydraulic control loading systems of flight simulators. Mechatronics, 38, pp.42-53.

[7] Sangpet, T. and Kuntanapreeda, S., (2013). Force control of an electrohydraulic actuator using a fractional-order controller. Asian Journal of Control, 15(3), pp.764-772. 
[8] Liem, D.T., Truong, D.Q., Park, H.G. and Ahn, K.K., (2016). A feedforward neural network fuzzy grey predictor-based controller for force control of an electro-hydraulic actuator. International Journal of Precision Engineering and Manufacturing, 17(3), pp.309-321.

[9] Liu, R. and Alleyne, A., 1999. Nonlinear force/pressure tracking of an electro-hydraulic actuator. IFAC Proceedings Volumes, 32(2), pp.952-957.

[10] Nakkarat, P. and Kuntanapreeda, S., (2009). Observer-based backstepping force control of an electrohydraulic actuator. Control Engineering Practice, 17(8), pp.895-902.

[11] Sun, H. and Chiu, G.C., (1999), June. Nonlinear observer based force control of electro-hydraulic actuators. In Proceedings of the 1999 American Control Conference (Cat. No. 99CH36251) (Vol. 2, pp. 764-768). IEEE.

[12] Baghestan, K., Rezaei, S.M., Talebi, H.A. and Zareinejad, M., (2014). Robust force control in a novel electro-hydraulic structure using polytopic uncertainty representation. ISA transactions, 53(6), pp.1873-1880.

[13] Rehman, W.U., Wang, S., Wang, X., Fan, L. and Shah, K.A., (2016). Motion synchronization in a dual redundant HA/EHA system by using a hybrid integrated intelligent control design. Chinese Journal of Aeronautics, 29(3), pp.789798

[14] Ferreira, J.A., Sun, P. and Grácio, J.J., (2006). Close loop control of a hydraulic press for springback analysis. Journal of Materials Processing Technology, 177(1-3), pp.377-381

[15] Yao, J., Wang, P., Cao, X.M. and Wang, Z., (2018). Independent volume-in and volume-out control of an open circuit pump-controlled asymmetric cylinder system. Journal of Zhejiang University-SCIENCE A, 19(3), pp.203-210.

[16] Wu, F. and Prajna, S., (2004), June. A new solution approach to polynomial LPV system analysis and synthesis. In Proceedings of the 2004 American Control Conference (Vol. 2, pp. 1362-1367). IEEE.

[17] Hoshino, K., Sonoda, D. and Yoneyama, J., (2016). Design of dynamic output feedback laws based on sums of squares of polynomials. Nonlinear Systems-Design, Analysis, Estimation and Control.

[18] Aktas, A., Yazici, H. and Sever, M., (2019). LMI-based design of an I-PD+ PD type LPV state feedback controller for a gantry crane. Transactions of the Institute of Measurement and Control, 41(6), pp.1640-1655.

[19] Scherer, C., Gahinet, P. and Chilali, M., (1996). $\mathrm{H} \infty$ design with pole placement constraints: An LMI approach. IEEE Transactions on Automatic Control, 41(3), pp.358-367.

[20] Wu, F. and Prajna, S., (2005). SOS-based solution approach to polynomial LPV system analysis and synthesis problems. International Journal of Control, 78(8), pp.600-611.

[21] Chesi, G., (2010). LMI techniques for optimization over polynomials in control: a survey. IEEE Transactions on Automatic Control, 55(11), pp.2500-2510.

[22] Prajna, S., Papachristodoulou, A. and Wu, F., 2004, July. Nonlinear control synthesis by sum of squares optimization: A Lyapunov-based approach. In 2004 5th Asian Control Conference (IEEE Cat. No. 04EX904) (Vol. 1, pp. 157-165). IEEE.

[23] Henrion, D. and Garulli, A. eds., 2005. Positive polynomials in control (Vol. 312). Springer Science \& Business Media 